\title{
Adding prostate-specific membrane antigen positron emission tomography (PSMA PET) to our prostate cancer armamentarium raises many questions
}

\author{
Laurence Klotz, CM, MD \\ Sunnybrook Hospital, University of Toronto, Toronto, ON, Canada
}

Cite as: Klotz L. Adding prostate-specific membrane antigen positron emission tomography (PSMA PET) to our prostate cancer armamentarium raises many questions Can Urol Assoc J 2021;15(6): 179-80. http://dx.doi.org/10.5489/cuaj.7336

See related paper on page 173

$\mathrm{P}$ rostate-specific membrane antigen positron emission tomography (PSMA PET) is a major advance in detecting recurrent prostate cancer and is increasingly used for prostate cancer diagnostics. PSMA is a transmembrane protein that provides an important target for radiolabeled imaging, since its expression is upregulated in malignant prostate cells, correlates with higher tumor grades, and is negatively regulated by androgen levels (increased expression with castration). According to these features, it is near ideal for detecting significant recurrences, both primary and in the castration-resistant state.

Several PSMA tracers have been used. The first ligand was a monoclonal antibody directed against PSMA (Prostascint). The limitation was that the recognized segment of the antigen was intracellular, resulting in high false-negative rates. More recently, small-molecule ligands that bind specifically to PSMA have been developed. There are now more than six such different ligands; these can be labelled by F, Ga, or Lutetium. ${ }^{18} \mathrm{~F}$-DCFPyL is a second-generation ${ }^{18} \mathrm{~F}$-labeled PSMA-targeted radiotracer. ${ }^{1}{ }^{18} \mathrm{~F}$-DCFPyL has shown higher tumor-to-background ratios compared to ${ }^{68} \mathrm{Ga}$-PSMA. ${ }^{2}$ The ${ }^{18} \mathrm{~F}$-radionuclide seems to provide better PET image resolution than ${ }^{68} \mathrm{Ga}$ due to a shorter positron range and higher positron yield, which may improve early detection of small metastases. It also has a longer half-life (110 vs. 68 minutes), allowing for more convenient preparation and administration.

The advent of a more sensitive imaging modality leads to stage migration, as patients previously assessed as being lower-stage are re-categorized into higher-stage disease. The pitfall of stage migration is that because the patients represent favorable disease compared to others in their new stage grouping, the apparent survival in each stage improves, even if there is no impact on individual patient outcome. Hence, important questions arise regarding this new modality. Does earlier identification of the site of recurrent disease translate into improved individual survival? Which is the best combination of radiotracer and ligand? Which patients should have the test, and how should the results be translated into patient management? What is the benefit of salvage therapy for oligometastatic disease identified on PSMA PET, and what is the optimal salvage therapy?

In the study by Chaussé et al in this issue of $(U A),{ }^{3}$ the authors sought to compare the performance of ${ }^{18} \mathrm{~F}$-DCFPyL PSMA PET to conventional imaging (bone scan and computed tomography $[\mathrm{CT}]$ ) with respect to identifying recurrent disease, and to determine how often this led to a change in management. Ninety-three patients were evaluated, with a median prostate-specific antigen (PSA) of 2.3. The study was strongly positive; $82 \%$ of patients were found to have a site of disease recurrence, conventional imaging was only $20 \%$ accurate, and $44 \%$ of patients were judged to have their management changed. Local recurrence was identified in $21 \%$ of patients following prostatectomy vs. $52 \%$ postirradiation (6/28 vs. $13 / 25$ patients).

Whether a change in management occurred was determined by a group of expert reviewers retrospectively rather than by the physician treating the patient at the time. This methodology is prone to bias. Prospective point-of-care data (currently being collected in the PREP study led by Glen Baumann) will provide more reliable data on this important question.

A further point of controversy currently is the value of the local treatment of oligometastases, most commonly with stereotactic body radiotherapy. Studies to date of metastasisdirected radiotherapy demonstrate high rates of local control and a small proportion of patients without progressive disease after two years. This approach appears to delay the requirement for androgen deprivation therapy; whether it will translate into improved cancer-specific survival remains to be determined. This is currently being evaluated by several randomized, prospective trials. 
Klotz

The authors assumed that PSMA PET-positive lesions were true-positives. Rigorous verification of PSMA PET-detected lesions is seldom performed, often because histological verification (biopsy) of small lesions is not feasible or not considered clinically appropriate. In practice, PSMA PETdetected lesions that are also seen on conventional imaging are assumed to be true-positives. Since the presence of PSMA-avid lesions may lead to the initiation of treatment, false-positive lesion detection could lead to overtreatment. Some studies have estimated a $20-30 \%$ false-positive rate, most commonly as local recurrence in the irradiated prostate. I suspect this is the reason for the relatively high rate of "local failure" in the radiation-treated patients in this study. Heavily irradiated, non-viable prostate cancer may remain PSMA-positive.

Clinical features are useful in identifying false-positives. The diagnostic certainty of detected lesions increases in the presence of characteristic abnormalities on CT, when peak standardized uptake value $\left(S U V_{\text {peak }}\right)$ is $\geq 3.5$, when PSA levels exceed $2.0 \mathrm{ng} / \mathrm{mL}$, or in patients with more than two PETpositive lesions. If one or more of these features is absent, a false-positive PSMA result should be suspected.
The incorporation of PSMA PET into our armamentarium raises as many questions as it addresses; this study by Chaussé et al moves the needle forward.

Competing interests: Dr. Klotz reports no competing personal or financial interests related to this work.

\section{References}

1. Gabriele D, Collura D, Oderda M, et al. Is there still a role for computed tomography and bone scintigraphy in prostate cancer staging? An analysis from the EUREKA-1 database. World I Urol 2016;34:517-23. https://doi.org/10.1007/s00345-015-1669-2

2. Perera $M$, Papa N, Roberts $M$, et al. Gallium-68 prostate-specific membrane antigen positron emission tomography in advanced prostate cancer-updated diagnostic utility, sensitivity, specificity, and distribution of prostate-specific membrane antigen-avid lesions: A systematic review and meta-analysis. Eur Uol 2020;77:403-17 https://doi.org/10.1016/i.eururo.2019.01.049

3. Chaussé G, Ben-Ezra N, Stoopler M, et al. Diagnostic performance of ${ }^{18 F-D C F P y L ~ p o s i t r o n ~ e m i s s i o n ~}$ tomography/computed tomography for biochemically recurrent prostate cancer and change-of-management analysis. Can Urol Assoc J 2021;15:172-7. http://dx.doi.org/10.5489/cuaj.6817

Correspondence: Dr. Laurence Klotz, Sunnybrook Hospital, University of Toronto, Toronto, ON, Canada; Laurence.Klotz@sunnybrook.ca

\section{CUA Practice Changing Publications (PCP)}

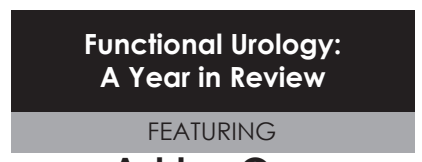

Ashley Cox \& Laura Nguyen
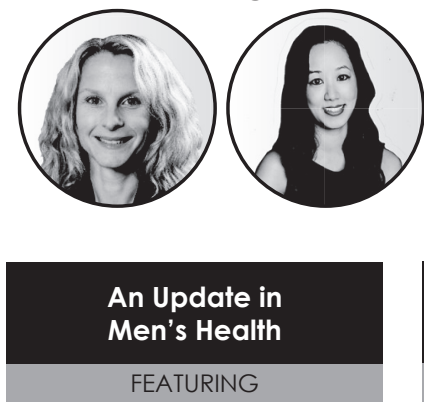
Jeffrey Campbell \& Premal Patel

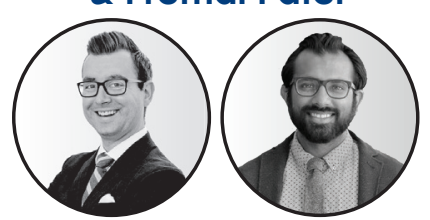

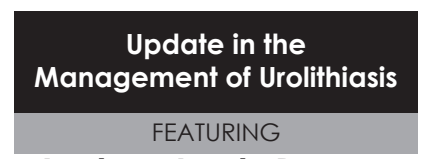

Andrea Lantz Powers \& Ben Chew
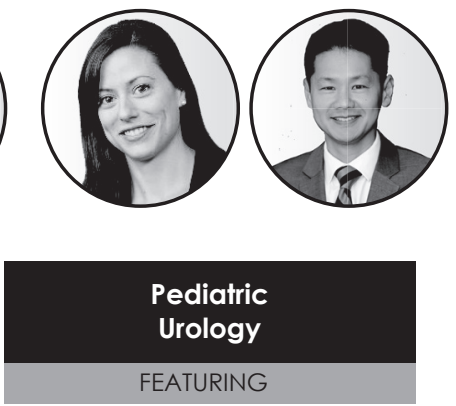

Melise Keays \& Peter Metcalfe

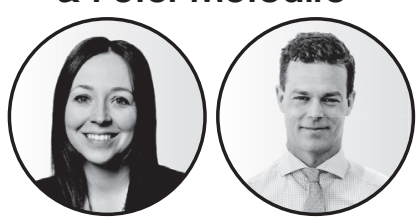

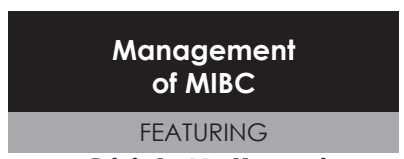

Girish Kulkarni

\& Ross Mason

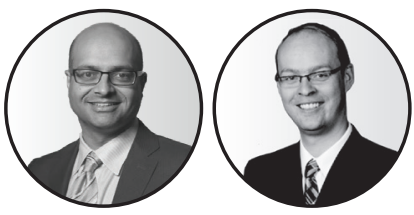

View each webinar and claim Section 3 credits any time on UROpedia Canada

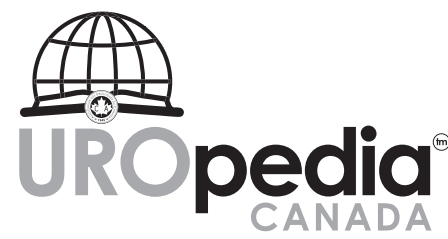

$\gg$ cua.org/UROpedia 